\title{
Application Article \\ Frequency Invariant Beam Steering for Short-Pulse Systems with a Rotman Lens
}

\author{
Andreas Lambrecht, Piotr Laskowski, Stefan Beer, and Thomas Zwick \\ Institute of High Frequency Techniques and Electronics, Karlsruhe Institute of Technology (KIT), Engesser Straße 5, \\ 76131 Karlsruhe, Germany \\ Correspondence should be addressed to Andreas Lambrecht, andreas.lambrecht@ihe.uka.de
}

Received 18 March 2010; Accepted 1 September 2010

Academic Editor: A. A. Kishk

Copyright ( $\odot 2010$ Andreas Lambrecht et al. This is an open access article distributed under the Creative Commons Attribution License, which permits unrestricted use, distribution, and reproduction in any medium, provided the original work is properly cited.

\begin{abstract}
A promising approach for beam steering of high-voltage transient signals for HPEM-systems (High Power Electro Magnetic) is presented. The inherent capability of the Rotman lens to provide true time delays is used to develop a prototype beam steering device for an antielectronics HPEM system in the frequency range from $350 \mathrm{MHz}$ to $5 \mathrm{GHz}$. Results of analytical calculations, simulations, and measurements from a hardware prototype are presented. The detailed mechanical setup of the Rotman lens is presented. Additionally the output pulses are investigated when inputting a Gaussian-like transient signal. Then time domain measures of quality (full width at half maximum, ringing, delay spread, maximum of transfer function) are investigated for these output transients, and the simulation and measurement results are compared. A concluding analysis of the realizable time domain array pattern shows the radiated pulse form.
\end{abstract}

\section{Introduction}

For the HPEM frequency range from 0.2 to $5 \mathrm{GHz}$ [1] beam scanning for high-voltage pulse signals over a large angle width is achieved best by using true time delay concepts for phasing of the antenna elements. The advantage of direct RF beamforming without up and down conversion is the low number of required switches compared to optical or digital IF beamfomers. Examples for RF beamformers are the Rotman lens [2], the Luneberg Lens [3], the Blass matrix [4], and switched RF lines [5]. In [6] an overview of these technologies is given. The main advantage of the Rotman and the Luneberg lenses is the low number of switching elements needed, compared to the Blass matrix and switched line concepts. The Rotman lens can feed a linear array, is capable of handling high-voltages, and is more compact than the Luneberg lens. Consequently it is attractive for RF-systems needing wide angle scanning and wide bandwidth. This may also find applications in automotive radar systems $[7,8]$. The ray optical model of the Rotman lens is used with a proper analytical description of the port structures similar to [9]. The input and output ports and the dummy region of the parallel plate region are the main restrictions to the usable bandwidth [10]. Although the Rotman lens is inherently broadband, most publications use it as narrowband. Additional to the treatment in the spectral domain, the lens is investigated in the time domain, too, which was, as far as the authors know, only done in one paper ever [11]. The couplings of the feeding lines and the interaction of all ports together are not straightforward to implement into the analytical system model, thus numerical field solvers are needed. The design of the dummy port region and the optimization of the whole Rotman lens are done numerically in CST Microwave Studio, where the initial model is based on the presented analytical design approach.

\section{True-Time-Delay Basics}

The treatment of antennas as Linear Time-Invariant systems is of special interest for the calculation of time domain responses of antenna arrays when inputting fast highvoltage transient signals. An antenna can be described in the time domain with its impulse response $h_{T x}(t, \theta, \psi)=$ 
IFFT $\left\{H_{T x}(f, \theta, \psi)\right\}$, where $H_{T x}$ is characterized by transmission measurements of two identical antennas [12]. For an ideal, passive power divider with adjustable true time delays $\tau_{\text {feed, } i}$, with $i=1, \ldots, N$ ( $N$ : number of antennas), the impulse responses are $h_{i}(t)=1 / \sqrt{N} \delta\left(t-\tau_{\text {feed, } i}\right)$. The antenna array is fed with a single pulse $v^{T x}(t)$. The superposition of the time delayed pulses in transmit mode at a far-field point gives the electrical field-strength of

$$
\begin{aligned}
e_{\text {total }}(t, \theta, \psi)= & \frac{1}{r} \frac{d}{d t} h^{T x}(t, \theta, \psi) * \sqrt{\frac{Z_{F 0}}{Z_{L 0}}} v^{T x}(t) \\
& * \underbrace{\sum_{i} h_{i}(t, \theta, \psi) * \delta\left(t-\frac{r_{i}}{c_{0}}\right)}_{f_{G r}^{+}},
\end{aligned}
$$

where $r$ is the distance to the far-field point, $\theta$ and $\phi$ are elevation and azimuth angle, respectively. $Z_{F 0}$ is the free space impedance, and $Z_{L 0}$ is the antenna input impedance. The operator “*” denotes a convolution. The sum describes the time domain array factor $f_{G r}^{+}$. The difference in the time delays between the antenna elements leads to a beam scanning angle $\beta$ of

$$
\beta=\arcsin \left(\frac{\Delta \tau c_{0}}{d_{z}}\right)
$$

where $c_{0}$ is the speed of light and $d_{z}$ is the element spacing in the antenna array, and $\Delta \tau=\tau_{\text {feed, } i}-\tau_{\text {feed, } j}$.

The $\tau_{\text {feed, } i}=\sqrt{\varepsilon_{r}} D_{i} / c_{0}$ are realized in the parallel plate region of the Rotman lens between the beam ports and the array ports:

$$
D_{i}=\sqrt{\left(X_{\mathrm{AP}, i}-X_{\mathrm{BP}, i}\right)^{2}+\left(Y_{\mathrm{AP}, i}-Y_{\mathrm{BP}, i}\right)^{2}}+W_{i} .
$$

The $X_{\mathrm{BP}, \mathrm{AP}}$ and $Y_{\mathrm{BP}, \mathrm{AP}}$ are the coordinates of the beam andarray ports on the contours of the Rotman lens.

The frequency response of the complete system is synthesized from the directional frequency responses of the antenna $(T x)$ and the frequency response of the beamformer $(\mathrm{BF})$, where the last one is directly connected with the time domain array factor $f_{\mathrm{gr}}(t, \theta, \psi)=h_{\mathrm{BF}, i}(t, \theta, \psi)$.

The following equation is valid for eachbeam port $i$ :

$$
H_{\text {ges }}\left(f, \theta_{i}, \psi\right)=H_{T x}\left(f, \theta_{i}, \psi\right) * H_{\mathrm{BF}, i}(f) .
$$

\section{Design Model for the Rotman Lens}

The design variables used in the following can be found in Figure 1. The lens is etched on a substrate with permittivity $\varepsilon_{r}$; for the microstrip lines this changes to $\varepsilon_{r, \text { eff. The }}$ procedure to obtain the coordinates of the array ports $P(x, y)$ and the lengths of the connecting lines $W$ to the linear antenna array $Q(N)$ by equating the path lengths in the lens can be found in many publications $[2,13]$. The three focal points $F_{1}, G, F_{2}$ on the beamport arc lead to zero phase error, but to realize more beam directions more input points on a connecting line are needed. This connecting

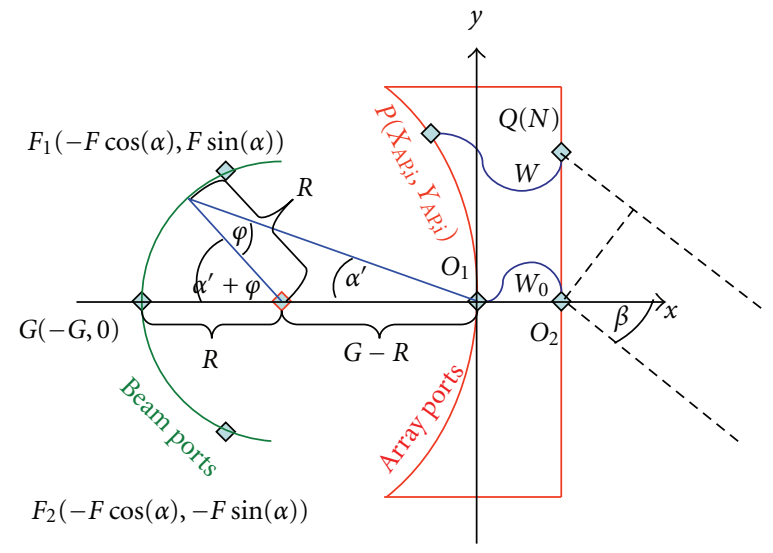

FIgure 1: Geometrical parameters of Rotman lens.

line can be realized by an elliptical focal arc. The phase center location is derived by using tangential lines onto the exponential curvature (Figure 2(b)) of the microstrip tapered ports, which connect to the parallel plate region. The parallel plate region can be seen as a 2-dimensional free space, since only TEM-waves can propagate. Therefore the ports can be described as 2-dimensional antennas with a 1dimensional radiating edge (Figure 2(a)). Following [14] the 2-dimensional propagation can be modeled with a modified Friis formula, with $r$ the distance between the center points of the radiating edge ports $L_{b, a}$ with the radiation characteristic of the microstrip port $F_{\text {port }}(\phi)_{b, a}$ (see Figure $2(a)$ ), and $\lambda$ the wavelength

$$
\frac{P_{r}}{P_{t}}=\frac{L_{a} L_{b}}{\lambda r} F_{\text {port }}^{2}(\phi)_{b} F_{\text {port }}^{2}(\phi)_{a} .
$$

$P_{t}$ describes the power put into the parallel plate region and $P_{r}$ the power received at one array port. The ports are orthogonal to the parallel plate region and can radiate into the angle range of $-\pi / 2 \ldots \pi / 2$. For the reflection coefficient the following relationship with the radiation characteristic of an edge port holds:

$$
r_{\text {port }}=\sqrt{1-\frac{L_{b, a}}{\lambda} \int_{-\pi / 2}^{\pi / 2} F_{\text {port }}^{2}(\phi)_{b, a} d \phi} .
$$

The radiation characteristic (see Figure 2(a)), of a microstrip port can be approximated by:

$$
F_{\text {port }}(\phi)_{b, a}=\frac{\sin \left(\left(\pi L_{b, a} / \lambda\right) \sin (\phi)\right)}{\left(\pi L_{b, a} / \lambda\right) \sin (\phi)} .
$$

The sidewall contours, connecting the beamport arc with the arrayport arc, have a triangular layout, see Figure 3. These contours are terminated with dummy ports, which absorb the incident power. This leads to certain restrictions for a good sidewall design. The dimensions of these dummyports are restricted by the same rules than the beam-/arrayports. To achieve an optimal angle of the dummy port to the incident wave it should point to the middle of the beam-/ arrayport contour. 


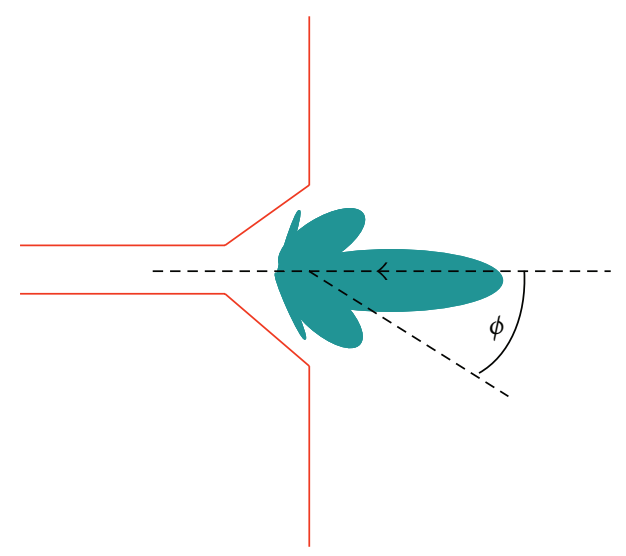

(a)

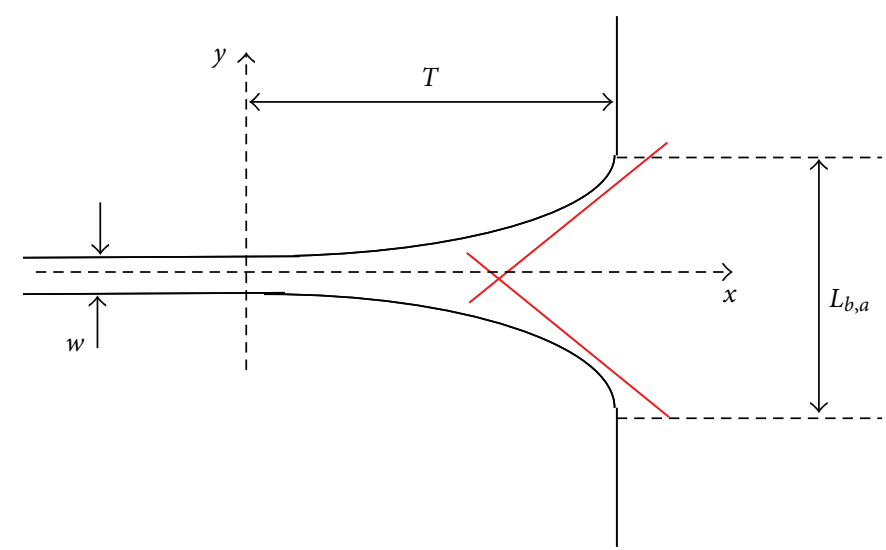

(b)

Figure 2: Port modelling. (a) Radiation pattern, (b) Phase center.

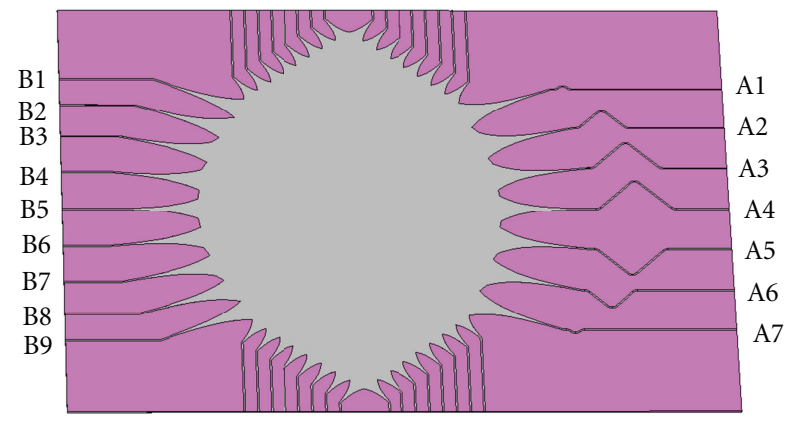

Figure 3: Simulation model of HPEM Rotman lens.

The simulation model for the HPEM-Rotman lens is fixed to a substrate size of $1219.2 \mathrm{~mm} \times 914.4 \mathrm{~mm}$. The size of the substrate is limited by the availability of substrate sheets and cost. The extension of the beam and array port contour in the shorter dimension is $508 \mathrm{~mm}$. The dummy port region extends only $153 \mathrm{~mm}$. The substrate is Arlon AD 1000 with a dielectric constant of 10.2 and a dissipation factor of 0.0023 at $10 \mathrm{GHz}$.

\section{Simulation and Measurement Results}

The preliminary analytical modeling is developed to get the relevant geometrical design parameters of the Rotman lens. For the mechanical setup the simulation model in Figure 3 is split into two parts because the size of the lens exceeds available standard sizes of the substrate material Arlon AD 1000. The setup of the Rotman lens is shown in Figure 4. The two pieces are fabricated separately and are put together onto a metal plate, serving as a common ground. To prevent an air gap between the substrate silicon is applied between the substrates; see Figure 4(f). On the top side, the two substrate pieces are soldered along the lens structure. As $50 \Omega$ terminations to the dummyports RF power resistors $(\mathrm{CHF} 3523 \times \mathrm{NT}$ Series Power RF Chip Termination) are fixed between the dummyport line and the ground plane the array ports and the beamports are connected via $N$-connectors with flange mount onto the microstrip lines. The whole lens is fixed into a chassis box.

The $S$-parameters of the built Rotman lens are measured with a network analyzer (Rohde and Schwarz) and compared to the simulation results and the predictions of the analytical design approach. Two exemplary results are shown in Figure 5 for the transmission of the beamports $\mathrm{B} 1$ and $\mathrm{B} 2$ to the arrayport A2. The simulation result contains no losses. The prediction of the analytical approach for the behaviour of the transmission coefficient coincides with the measured results. Especially the breakdown of the transmission coefficient around $4 \mathrm{GHz}$ for $S_{\mathrm{A} 2 \mathrm{~B} 1}$ and around $4.5 \mathrm{GHz}$ for $S_{\mathrm{A} 2 \mathrm{~B} 1}$ is predicted well. This is due to the higher-order modes, that occur in the structure at higher frequencies. The simulation does not show this, due to restrictions to simulation time and thus to accuracy of the numerical field solver. Another reason for the degradation of the transmission by higher frequencies is the narrow directivity of the tapered ports with increasing frequency.

Since the system will be used for short transient signals, the received pulse at each antenna port (right side in Figures 3 and 9) is gated to neglect multipath reflections from inside the parallel plate region, thus giving the transmission loss for the main peak of the transient pulse. The transmission coefficient between beamport B5 and the arrayports A1, A3, A5, A7 is shown in Figure 7. The transmission to the outer arrayports gets worse with higher frequencies, leading to amplitude tapering, which will consequently lead to a loss in gain of the complete array. This is due to a higher directivity of the microstrip tapered ports with increasing frequency. The system will lose efficiency for spectral components with higher frequencies and for the outer beam ports. The phase of the transmission is perfectly linear over the whole frequency range of interest (Figure 8). The analytical model predicts a lower frequency bound of approximately $300 \mathrm{MHz}$, which is confirmed by the simulation result (Figure 6). The reflection coefficient of beamport B1 is a worst case beam port and the return loss stays below $-7 \mathrm{~dB}$ from $300 \mathrm{MHz}$ onward. 


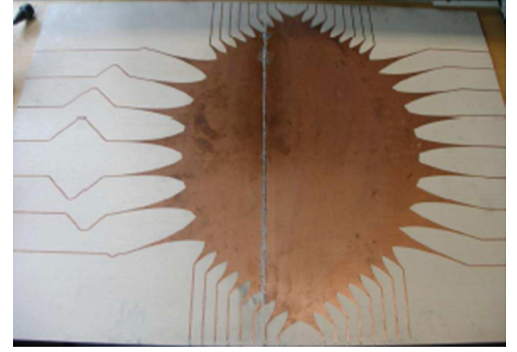

(a) Whole lens

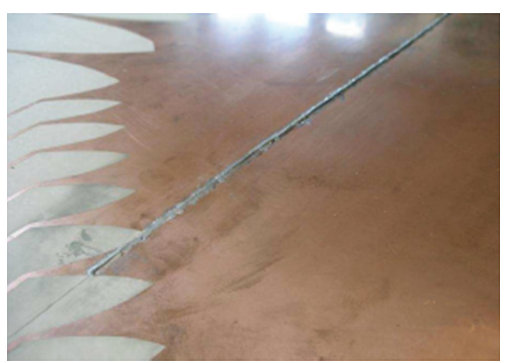

(b) Soldering

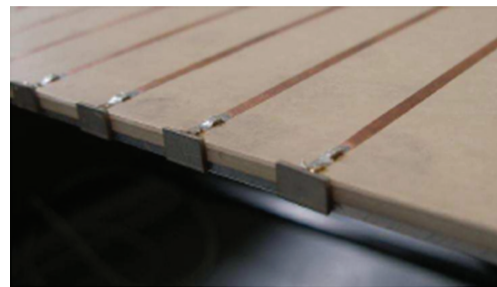

(c) Dummy resistors

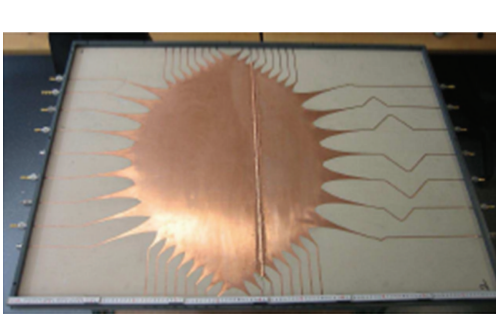

(d) Fixed into final chassis

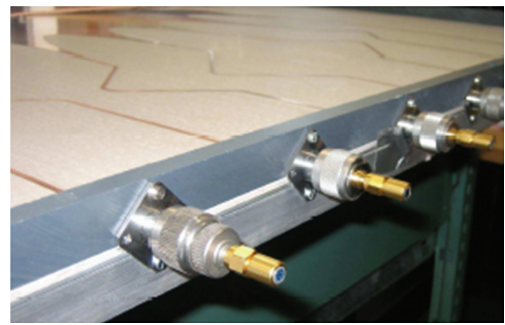

(e) $N$-connectors

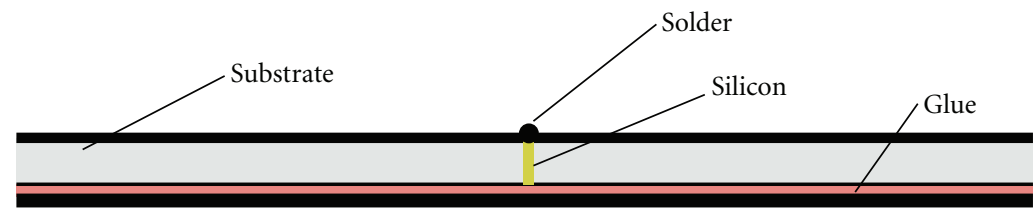

(f) Schematic setup

Figure 4: Mechanical setup of the Rotman lens.

\section{Measures of Quality for Transient Analysis}

There are two important effects to be distinguished: firstly, the ability of a system to effectively transmit power and secondly, the distorting influence on the waveform to be transmitted. The second effect is conveniently described with the system's time domain behaviour, thus the output pulses of the Rotman lens are investigated. In the following, $\left|h^{+}\right|$ denotes the absolute value of the envelope of the analytical signal of the signal $h$.

A measure for the linear distortion of the antenna is the envelope width, which is defined as the full width at half maximum (FWHM) of the magnitude of the responses envelope:

$$
\begin{array}{r}
\tau_{\mathrm{FWHM}}=t_{2}|| h^{+}\left(t_{2}\right)\left|=\max \left\{\left|h^{+}\right|\right\} / 2-t_{1}\right|\left|h^{+}\left(t_{1}\right)\right|=\max \left\{\left|h^{+}\right|\right\} / 2 \\
t_{2}>t_{1} .
\end{array}
$$

The duration of the ringing $\tau_{r, \alpha}$ is defined as the time until the envelope has fallen from the peak value below a certain lower bound, for example, a fraction $\alpha$ of the main peak

$$
\tau_{r, \alpha}=t_{2}|| h^{+}\left(t_{2}\right)\left|=\alpha \cdot \max \left\{\left|h^{+}\right|\right\}-t_{1}\right|\left|h^{+}\left(t_{1}\right)\right|=\max \left\{\left|h^{+}\right|\right\} .
$$

A reasonable-value of $\alpha$ is given in [11] as 0.22 .

An integral parameter describing the dispersive properties of a system in the time domain is the delay spread $\tau_{\mathrm{DS}}$ of the real valued transient response. The delay spread is calculated from the power related $\left|h_{n}\right|^{2}$

$$
\tau_{\mathrm{DS}}(\theta, \psi)=\frac{\int_{-\infty}^{\infty}\left(t-\tau_{D, \text { mean }}(\theta, \psi)\right)\left|h_{i, \mathrm{copol}}(t, \theta, \psi)\right|^{2} d t}{\int_{-\infty}^{\infty}\left|h_{i, \mathrm{copol}}(t, \theta, \psi)\right|^{2} d t}
$$

with

$$
\tau_{\text {DS,mean }}(\theta, \psi)=\frac{\int_{-\infty}^{\infty} t\left|h_{i, \text { copol }}(t, \theta, \psi)\right|^{2} d t}{\int_{-\infty}^{\infty}\left|h_{i, \text { copol }}(t, \theta, \psi)\right|^{2} d t}
$$

The maximum value of the absolute value of the envelope of the impulse response is important for the achievable signal strength at the output port:

$$
h_{\max }=\max _{t}\left|h_{c o}\right|
$$

These measures of quality can now be used to quantify the transient behaviour of the Rotman lens.

\section{Time Domain Results}

In Figure 9 the measured output transient pulses at four arrayports (A1, A2, A3, A4) when feeding beamport B5 


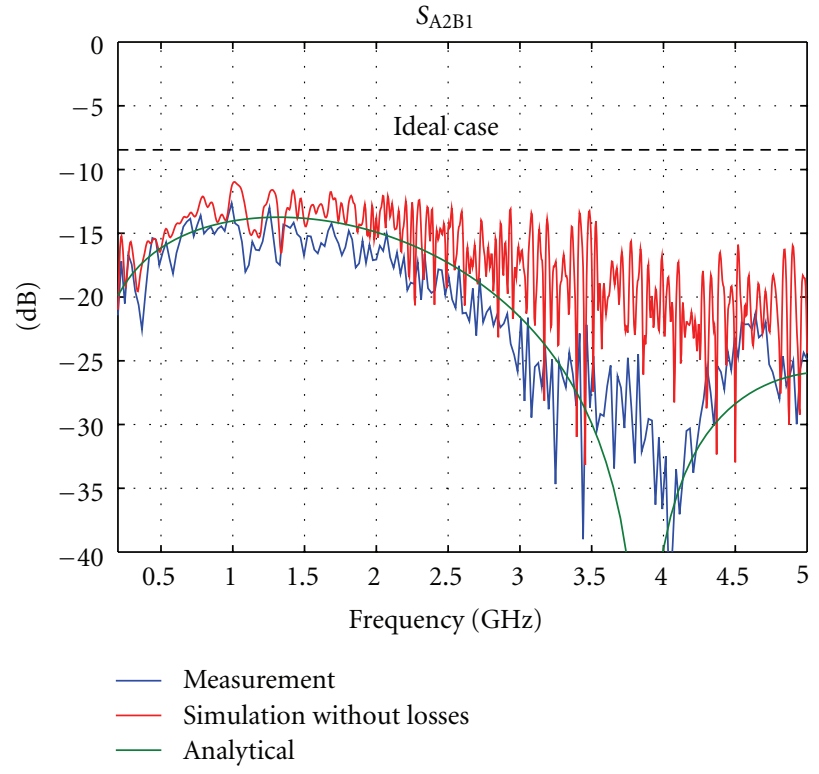

(a)

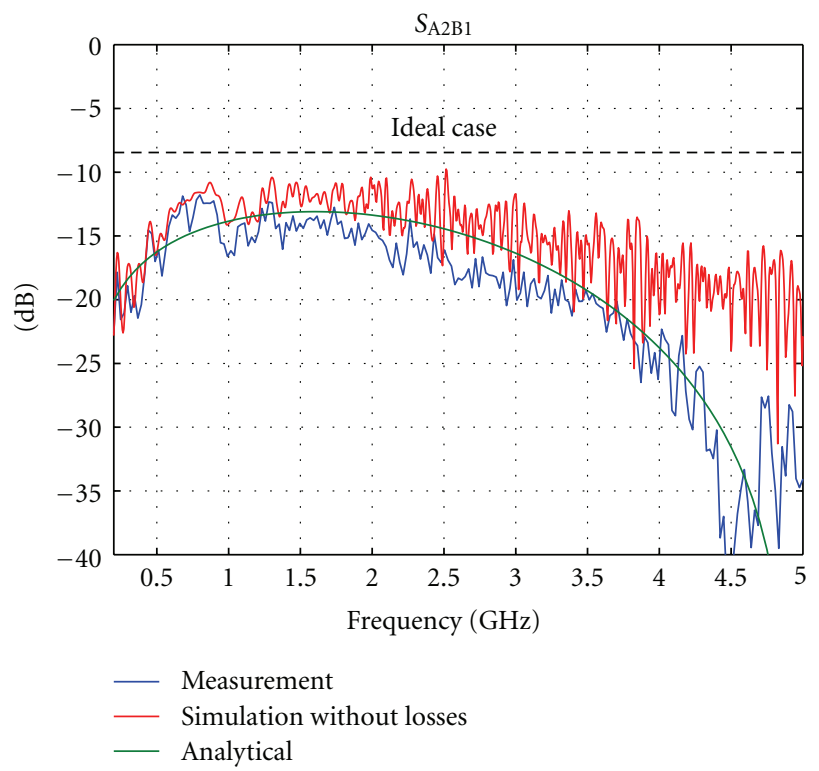

(b)

FIgURE 5: Comparison of transmission coefficients: analytical model, simulation, and measurement.

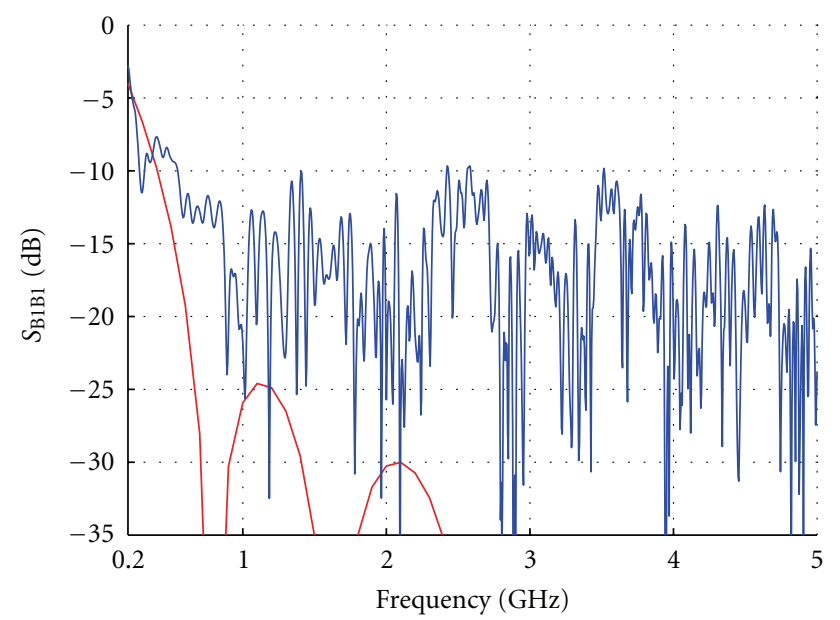

- Analytical

- Simulation

Figure 6: Comparison of reflection coefficient between the analytical model and simulation.

are shown. A Picosecond Puls Generator 3600 is used as input waveform generator. The input waveform is a Gaussian-like inverted voltage pulse, When looking at the output voltage pulse one can see a similarity with a first derivative of a Gaussian monocycle transient pulse plus some additional ringing due to multipath and internal reflections inside the Rotman lens. Thus one can conclude that an ultrawideband pulse undergoes a derivation when transmitted through the lens. This derivation occurs in the tapered ports of the structure, which are the only place where the passive Rotman lens can differentiate.

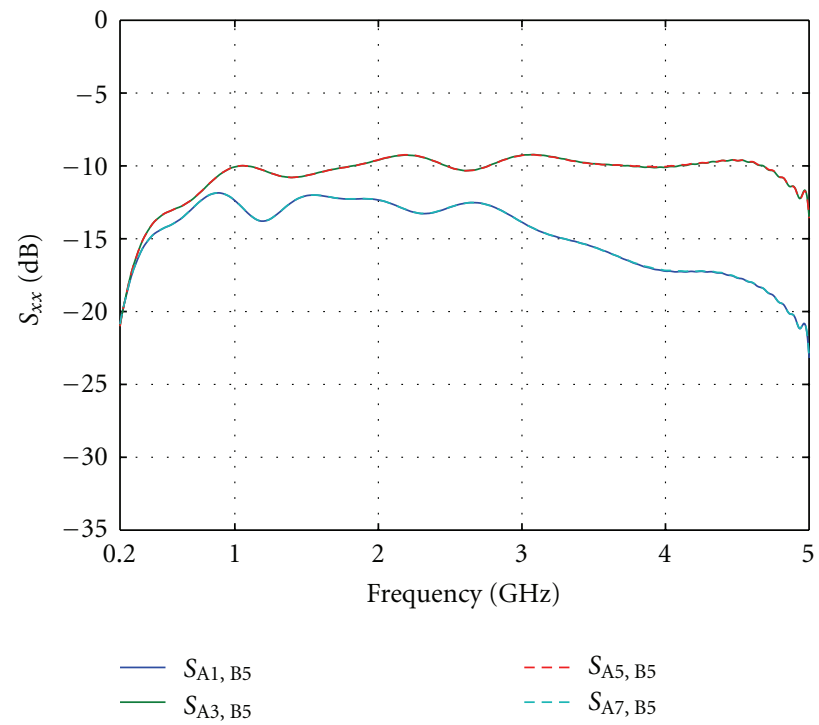

Figure 7: Amplitude of transmission after gating the main pulse, Input at B5.

The measures of quality are shown in Figures 10, 11, 12 and 13. What all these figures have in common is that the simulation results are much smoother than the measurement results. It is expected that beamport B5 performs best because for this beamport the structure is completely symmetrical. This is confirmed by the measurements but could not be predicted so clearly by simulation (see, e.g., Figure 10). The measured FWHM for B5 is between 1 ns and $1.2 \mathrm{~ns}$. The ringing of the output pulses for $\mathrm{B} 5$ is between $0.8 \mathrm{~ns}$ and $1 \mathrm{~ns}$. Following [15], a UWB-HPEM transient pulse has a pulse length of $2.5 \mathrm{~ns}$. Thus when inputting 


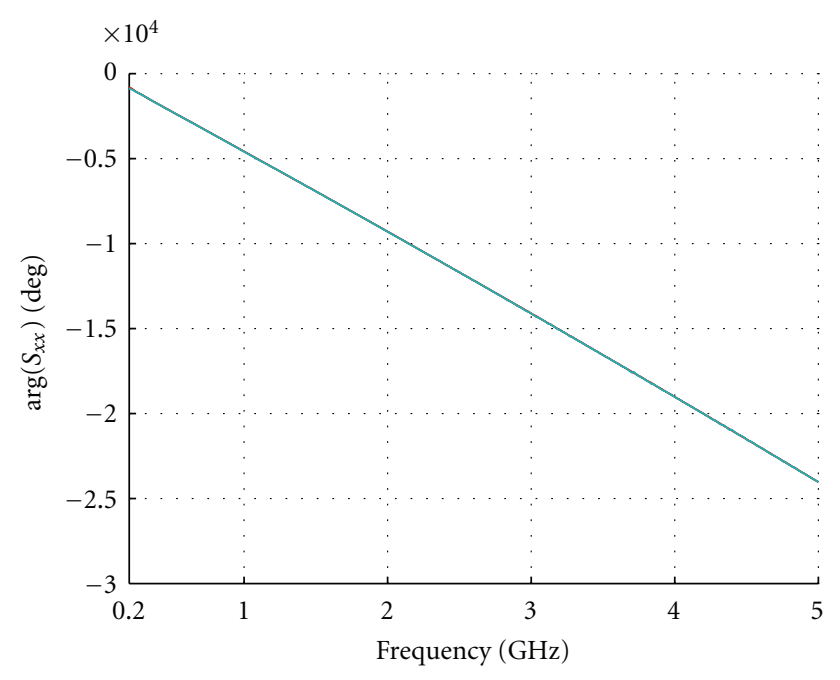

Figure 8: Phase of transmission. Input at B5. Output at A1, A3, A5, and A7.

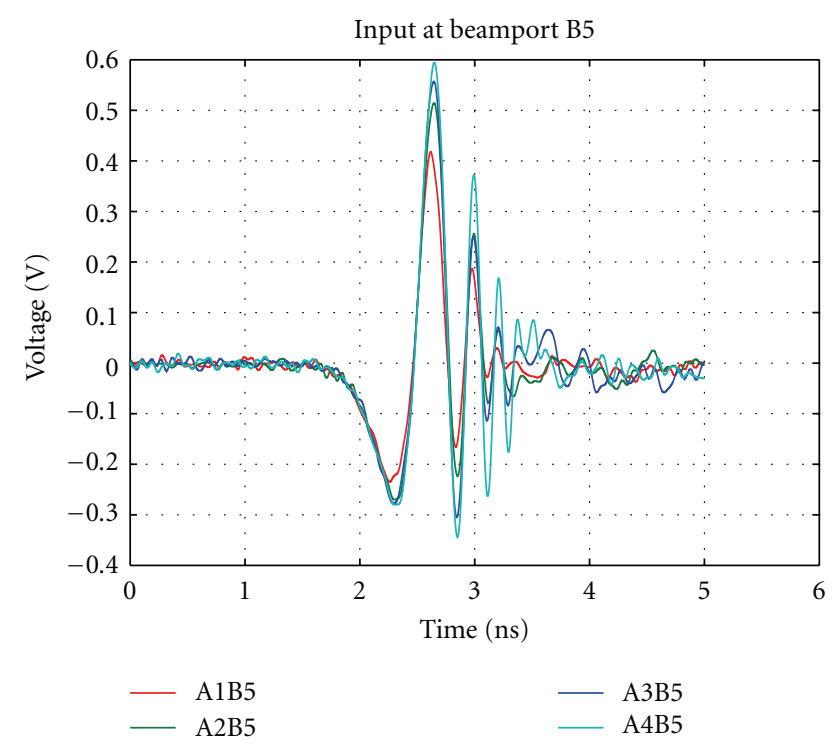

Figure 9: Measured output transient pulses of the Rotman lens for feeding B5.

a proper HPEM pulse into the lens, this pulse should not exceed an FWHM of approximately $1 \mathrm{~ns}$ because the lens will add around 1 to 1.2 ns to this pulse width.

The delay spread calculated from the measurements varies much more than that for the simulations hinting at more dispersion in the real lens than could be considered in simulations. This is worsened by the split up of the substrate in two pieces due to the mechanical setup constrains.

The distribution of the maximum of the transfer function of the lens shows the amplitude tapering across the arrayports due to the directivity of the ports. Simulations predicted a higher $h_{\max }$ for all ports due to the absence of metallic and dielectric losses. The measurement results for B5 should ideally be symmetric like the simulation result but

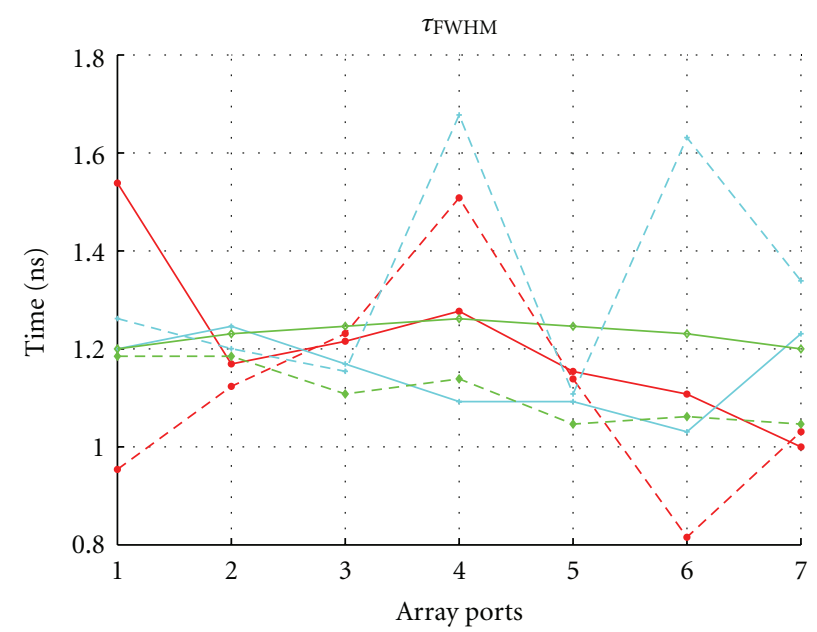

FIGURE 10: Full width at half maximum (FWHM): Simulation and measurement.

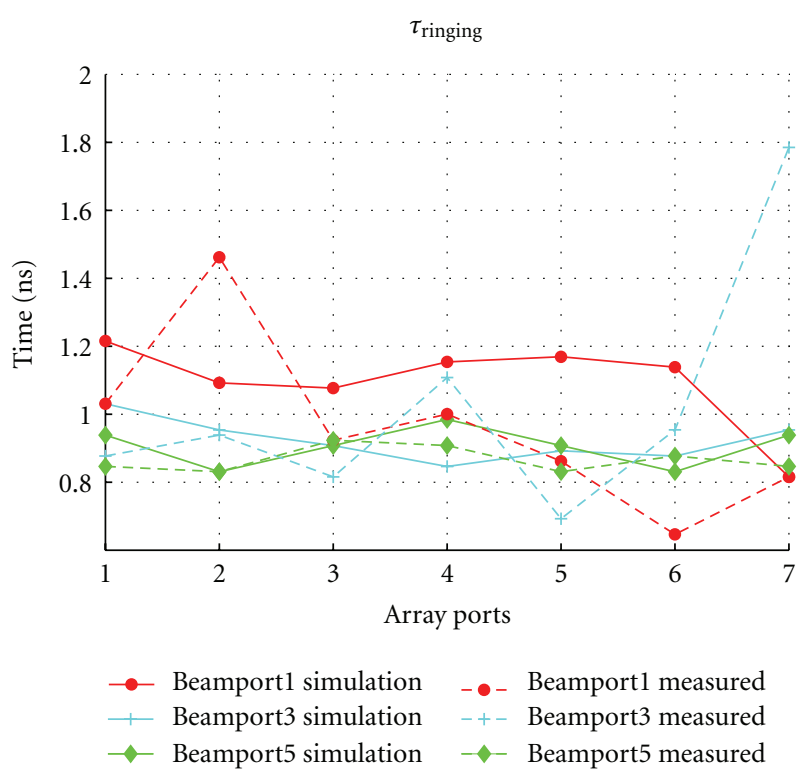

FIGURE 11: Output ringing time: Simulation and measurement.

due to the mechanical setup this has not been expected. But the measurements are symmetric, indeed.

Overall simulation results and measurements confirm the suitability of the Rotman lens to beam steer high-voltage transient signals. Input transients will be broadened, and dispersion will influence the efficiency. This is confirmed because the amplitudes of $h_{\max }$ are much lower than expected from the lossless and dispersion-free simulations.

The $S$-parameters are used to synthesize the array factor of a $7 \times 1$ antenna array with isotropic radiators; see Figure 14. The beam is stationary over the whole frequency range. Since the system uses transient signals the impulse response of this array gives a better impression of the performance of the frequency invariant beam steering. In 


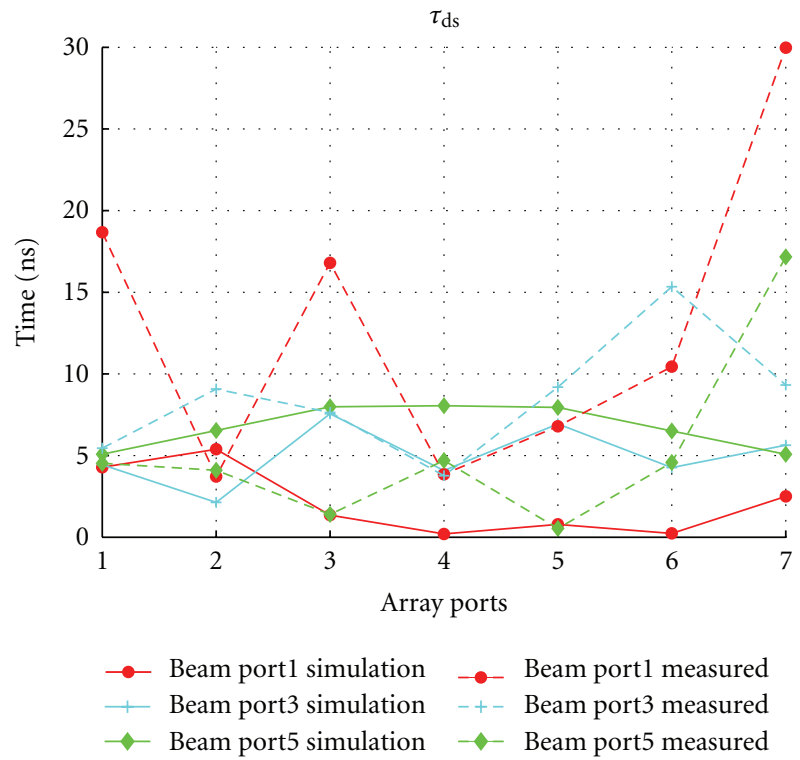

Figure 12: Output delay spread: simulation and measurement.

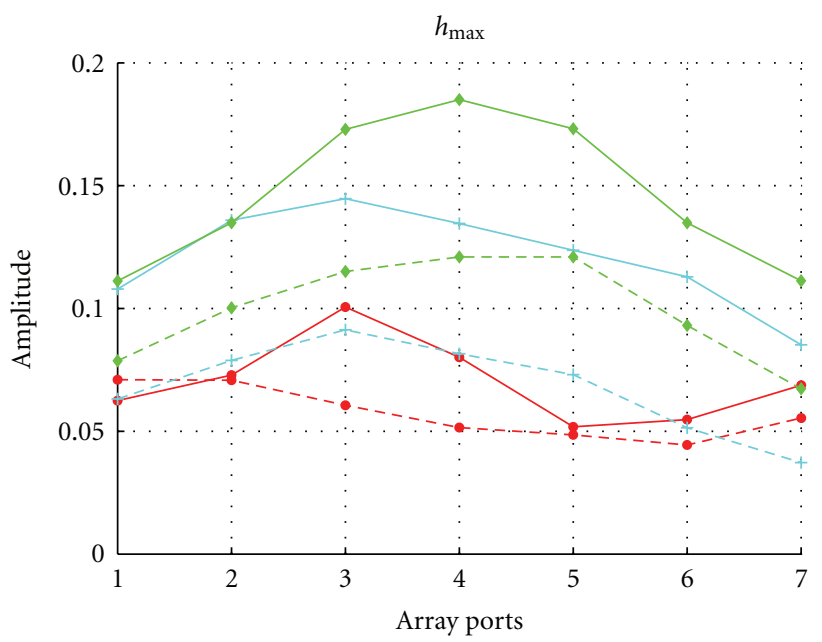

FIGURE 13: Output maximum transfer function: simulation and measurement.

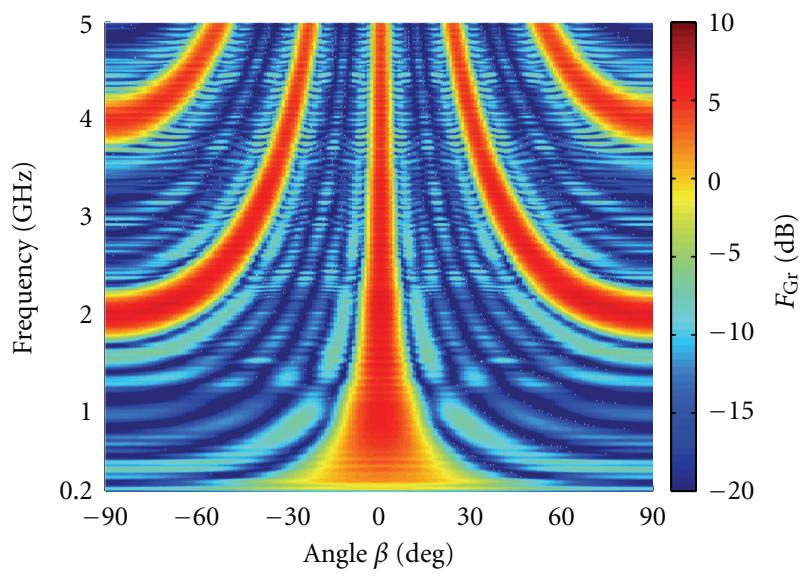

FIGURE 14: Gain pattern of synthesised antenna array.

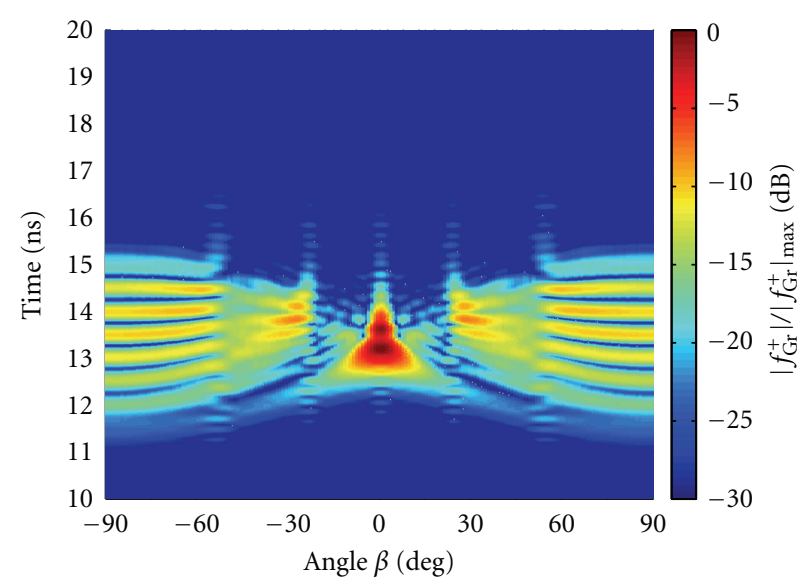

FIGURE 15: Impulse response of synthesised antenna array.

Figure 15 the time domain array factor is plotted normalized to its maximum. No gating of the $S$-parameter was applied, and a second pulse maximum after the main peak is visible like in Figure 9. From the figure one can clearly confirm the results from Figure 10, that is, a pulse width of approximately slightly above $1 \mathrm{~ns}$. The dispersion of the main peak gets stronger for the outer beam ports. This is the first time a Rotman lens is characterized with its time domain behavior for an HPEM-system.

\section{Conclusion}

The concept of beam steering of transient high-voltage signals with a Rotman lens gives promising results for a possible implementation in an HPEM-system. The transient input signals can have voltages up to over $10 \mathrm{kV}$, and there is no need for synchronization of sources. Since efficiency is only between $10 \%$ and $60 \%$, this concept is only useful when influencing electronics in small distances to the array $(<100 \mathrm{~m})$. It was possible to realize a lens which gave good performance results between $350 \mathrm{MHz}$ and $5 \mathrm{GHz}$.

The measurement results give detailed information on the expected pulse broadening by the lens when inputting Gaussian-like signals. A detailed analysis of the measures of quality FWHM, ringing, delay spread, and the maximum of the transfer function is given. The time domain array pattern confirms the results.

An interesting effect of the Rotman lens is shown by the measurements. The output pulses are not any more Gaussian pulses but look like first derivatives of a Gaussian pulse due to the differentiation behaviour of the tapered ports.

\section{Acknowledgment}

This paper is supported by Deutsche Forschungsgemeinschaft (DFG). 


\section{References}

[1] D. V. Giri and F. M. Tesche, "Classification of intentional electromagnetic environments (IEME)," IEEE Transactions on Electromagnetic Compatibility, vol. 46, no. 3, pp. 322-328, 2004.

[2] W. Rotman, "Wide-angle microwave lens for line source applications," IEEE Transactions on Antennas and Propagation, pp. 623-632, 1963.

[3] R. K. Luneburg, "Mathematical theory of optics," American Journal of Physics, vol. 34, no. 1, pp. 80-81, 1966.

[4] J. Blass, "Multidirectional antenna-a new approach to stacked beams," IRE International Convention Record, vol. 8, part 1, pp. 48-50, 1960.

[5] J. Schoebel, "A true-time-delay phase shifter system for ultra-wideband applications," in Proceedings of the German Microwave Conference (GeMIC '08), Hamburg, Germany, March 2008.

[6] P. S. Hall and S. J. Vetterlein, "Review of radio frequency beamforming techniques for scanned and multiple beam antennas," IEE Proceedings H: Microwaves, Antennas and Propagation, vol. 137, no. 5, pp. 293-303, 1990.

[7] L. Hall, H. Hansen, and D. Abbott, "Rotman lens for mmwavelengths," in Smart Structures, Devices, and Systems, vol. 4935 of Proceedings of SPIE, pp. 215-221, 2002.

[8] J.-G. Lee, J.-H. Lee, and H.-S. Tae, "Design of a nonradiative dielectric Rotman lens in the millimeter wave frequency," in Proceedings of the International Microwave Symposium Digest IEEE-MTT-S, pp. 551-554, May 2001.

[9] M. S. Smith and A. K. S. Fong, "Amplitude performance of ruze and Rotman lenses," The Radio and Electronic Engineer, vol. 53, no. 9, pp. 329-336, 1983.

[10] E. O. Rausch and A. F. Peterson, "Rotman lens design issues," in Proceedings of the IEEE Antennas and Propagation Society International Symposium, pp. 35-38, July 2005.

[11] V. K. Tripp, J. E. Tehan, and C. W. White, "Characterization of the dispersion of a Rotman lens," in Proceedings of the IEEE Antennas and Propagation Society International Symposium (APS '89), pp. 667-670, June 1989.

[12] W. Sörgel, S. Knörzer, and W. Wiesbeck, "Measurement and Evaluation of Ultra Wideband Antennas for Communications," in Proceedings of the International ITG-Conference on Antennas (INICA '03), pp. 377-380, September 2003.

[13] T. Katagi, S. Mano, and S. Sato, "An improved design method of Rotman lens antennas," IEEE Transactions on Antennas and Propagation, vol. 32, no. 5, pp. 524-527, 1984.

[14] P. S. Simon, "Analysis and synthesis of Rotman lenses", in Proceedings of the 22nd AIAA International Communications Satellite Systems Conference and Exhibit (ICSSC '04), pp. 738748, May 2004.

[15] M. Camp and H. Garbe, "Susceptibility of personal computer systems to fast transient electromagnetic pulses," IEEE Transactions on Electromagnetic Compatibility, vol. 48, no. 4, pp. 829-833, 2006. 

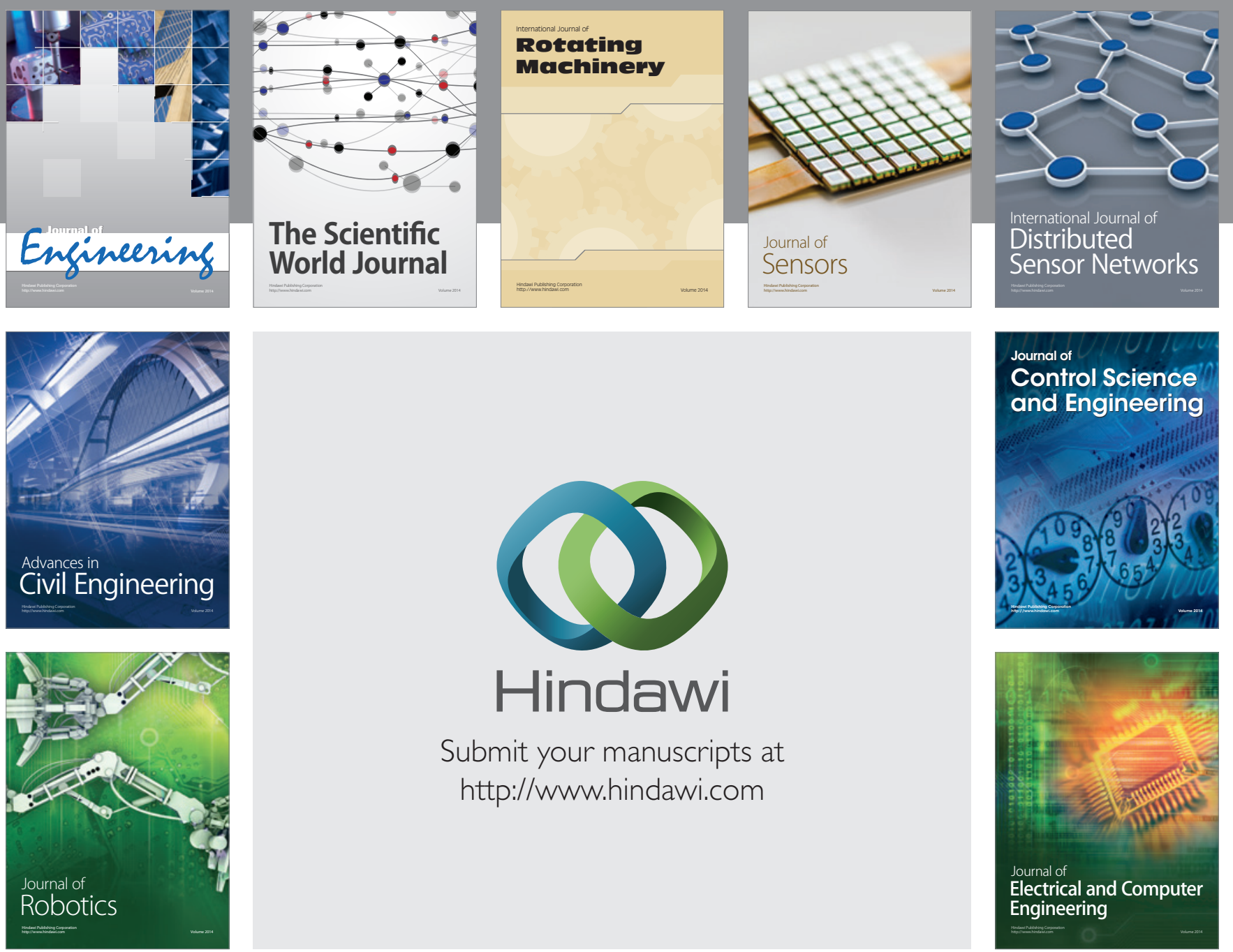

Submit your manuscripts at

http://www.hindawi.com
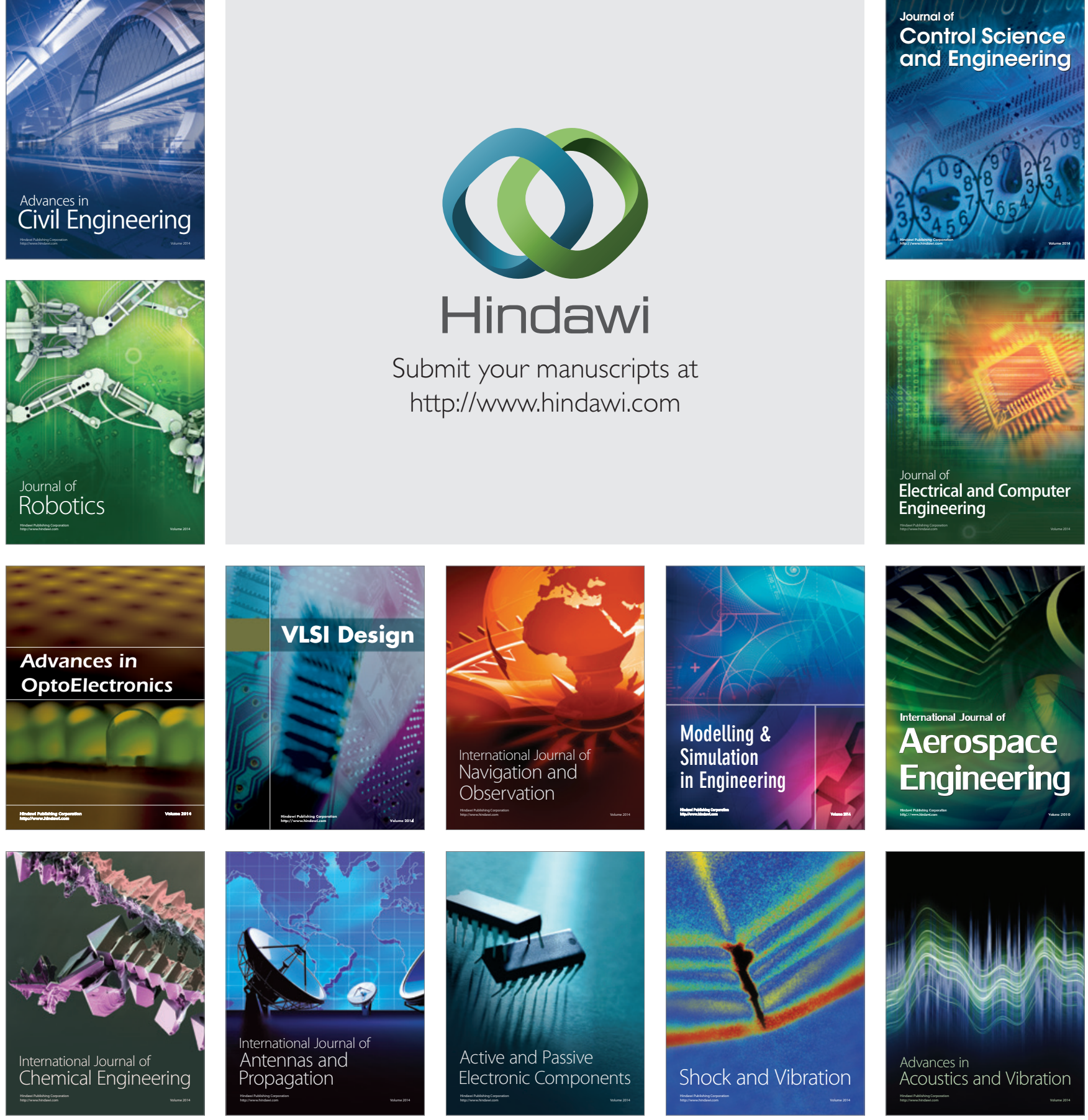